\title{
Invasive Bladder Cancer in the Eighties: Transurethral Resection or Cystectomy?
}

\author{
Oscar Rodriguez Faba, Joan Palou, Guillermo Urdaneta, Luis Gausa, Humberto Villavicencio
}

Department of Urology, Fundacio Puigvert, Universitat Autonoma de Barcelona, Barcelona, Spain

\begin{abstract}
Purpose: Describe morbidity and survival in patients older than 80 years with muscle invasive bladder cancer (MIBC) treated with radical cystectomy (RC) or transurethral resection (TUR) in our institution.

Materials and Methods: We reviewed our database of all patients older than 80 years treated with RC and TUR for MIBC between 1993 and 2005 in our institution. Twenty-seven patients were submitted to RC, with mean age of 82 years and mean follow-up of 16.4 months. RC was carried out following diagnosis of previous MIBC in 14 cases (51.9\%). The American Society of Anesthesiology (ASA) score was III or IV in 23 patients (85.1\%). Seventy-two patients with a mean age of 84 years and mean follow-up of 33 months, diagnosed with MIBC, were managed by means of TUR. The ASA score was III-IV in $64(88.8 \%)$ patients.

Results: Pathological stage of the RC specimen was pT3 in 18 cases (66.7\%). Mean hospital stay was 16 days. Early complications were assessed in 8 patients (29.6\%), with an overall survival (OS) of 42.94\%, and cancer-specific survival (CSS) of $60.54 \%$. In patients submitted to TUR, clinical stage was T2 in 36 cases (50\%). The mean hospital stay was 7 days, with a readmission rate (RR) of $87.5 \%$. OS and CSS was less than $20 \%$.

Conclusions: RC in octogenarian patients is a safe procedure, with complication and survival rates comparable to RC series in general population. Transurethral resection (TUR) for patients with MIBC within this age range is a much less morbid procedure, but disease specific survival is lower.
\end{abstract}

Key words: urinary bladder neoplasms; cystectomy; endoscopy; aged

Int Braz. J Urol. 2011; 37: 49-56

\section{INTRODUCTION}

The increased incidence and prevalence of genitourinary neoplasias over the last few decades can be attributed to demographic evolution and increased life expectancy. The National Cancer Institute has estimated that $89 \%$ of the bladder tumors are found in patients older than 55, and that the mean age at diagnosis, is 73 years(1).

Standard treatment for muscle invasive bladder cancer (MIBC) is radical cystectomy with bilateral pelvic lymphadenectomy, and is justified in patients with life expectancy greater than two years. In patients more than 80 years old radical surgery tends to be avoided, since is believed to be associated with increased morbidity and mortality rates. Historically those patients have been treated with TUR that is a less aggressive procedure. It is now accepted that the American Society of Anesthesiology (ASA) score for patients undergoing a major procedure is a predictive factor for morbidity and mortality which is more significant than age alone (2). For the last seven 
years we have actively performed radical cystectomy (RC) in patients of advanced age with an ASA score compatible with this type of surgery.

\section{MATERIALS AND METHODS}

We reviewed our database of all patients that were treated with either RC or TUR between 1993 and 2005 for invasive bladder cancer and age over 80 that did not receive neoadjuvant or adjuvant therapy.

\section{Radical Surgery}

Twenty-seven patients over 80 years with MIBC were submitted to RC and urinary diversion at our institution between 1993 and 2005. Twentytwo $(81.5 \%)$ were males and $5(18.5 \%)$ females with mean age $82 \pm 3$ years (range 80 - 91 years). Inclusion criteria for the patients were detailed medical history, a physical examination, cardiovascular and pulmonary parameters including their metabolic and nutritional status. Indications of RC were based on finding MIBC in TUR, or recurrent high-grade superficial tumors unresponsive to Bacillus Calmette-Guerin (BCG) therapy. Before surgery all patients were staged according to the ASA score (3).

Following surgery patients were monitored at the post-operative care unit during 24 hours. Ureteral catheters were removed 7-10 days following cystectomy.

\section{Transurethral Resection}

A total of 72 patients (59 males and 13 females) over 80 years old were staged for MIBC and suitable for TUR. All cases were staged with bimanual examination under anesthesia and underwent TUR (complete or palliative). Multiple random biopsies were taken to document presence of carcinoma in situ. Follow-up consisted of cystoscopy and TUR if recurrence and/or progression occurred. During follow-up, the numbers of re-admittance and health state of the patients were considered to evaluate the progression of the disease.
Peri-operative mortality was defined as allcause death up to 30 days after surgery. The overall mortality rate included cancer-related or cancerunrelated deaths. The morbidity rate included early complications considered adverse effects in the course of hospitalization or 30 days post-surgery and late complications longer than 30 days postsurgery.

\section{RESULTS}

\section{Cystectomy}

Pathology of radical cystectomy (RC) revealed urothelial carcinoma in 26 of the 27 patients (96.3\%). Stage was pT3 in 18 cases (66.7\%) and association with prostate carcinoma was found in 10 cases $(37 \%)$ (Table-1).

Urinary diversion was ileal conduit in 24 $(88.8 \%)$ patients, cutaneous diversion in two patients $(7.40 \%)$ and Studer orthotopic substitution in one patient $(3.80 \%)$.

Concomitant cardiovascular disease was the most frequent co-morbidity, with hypertension in 10 patients $(37 \%)$, previous acute myocardial infarction in 3 patients $(11.1 \%)$ and arrhythmia in 2 patients (7.4\%). Other concomitant pathologies were diabetes mellitus in 4 patients (14.8\%), bronchial asthma in 1 patient $(3.7 \%)$ and emphysema in 3 patients $(11.1 \%)$.

Intra- or post-operative blood transfusion was necessary in 10 patients $(37 \%)$; furthermore there were two cases $(7.4 \%)$ of intraoperative mortality. Early complications occurred in 8 patients $(29.6 \%)$ and were paralytic ileus in 4 patients, wound infection in 3, intra-abdominal abscess in 1 patient and entero-cutaneous fistula in another one. The major complications were: acute myocardial infarction in one patient and another patient require re-operation due to bleeding.

The mean hospital stay was 16 days (8-28) and the mean follow-up of these patients was 16.4 months (1.4-84.2 months).

Distant disease was found in 8 patients during follow-up: four of those diseases involved lymph nodes and 4 were multiple. 
Table 1- Patient characteristics.

\begin{tabular}{cccc}
\hline & Patient Characteristics & Cystectomy (\%) & TUR (\%) \\
\hline Number of patients & Male & 27 & 72 \\
\multicolumn{1}{c}{ Sex } & Female & 22 & 59 \\
& II & 5 & 13 \\
\multirow{2}{*}{ ASA (\%) } & III & $4(14.8)$ & 0 \\
& IV & $19(70.4)$ & $20(27.7)$ \\
& pT0 & $4(14.8)$ & $44(61.1)$ \\
& pT2/cT2 & $2(7.4)$ & $36(50)$ \\
Stage (\%) & cT2-T3 & $3(11.1)$ & $24(33.3)$ \\
& pT3/cT3 & & $10(13.9)$ \\
& pT4/cT4 & $18(66.7)$ & $2(2.8)$ \\
& Squamous & $4(14.8)$ & \\
\hline
\end{tabular}

ASA = American Society of Anesthesiology score; $T U R=$ transurethral resection.

Overall survival (OS) was $42.94 \%$ and cancer specific survival (CSS) $60.54 \%$ (Table-2, Figure-1).

\section{Transurethral Resection}

In patients submitted to TUR, the stage was cT2 in 36 cases (50\%), cT3 in 10 cases (13.9\%), cT2$\mathrm{T} 3$ in 24 cases $(33.3 \%)$, and cT4 in 2 cases $(2.8 \%)$. Grade was G3 in 49 cases (68\%). Forty-nine patients $(68 \%)$ had a complete TUR, whereas in $32 \%$ resection was incomplete (Table-1).

Intra- or post-operative blood transfusion was necessary in 10 patients $(13.8 \%)$, and there was no case of intra-operative death. Regarding early complications, hemostatic intervention was required in 6 patients (8.3\%), and 2 patients showed manifestations of hemodynamic angina that required intensive care. The mean hospital stay was 7 days; with a readmission rate of $87.5 \%$ (thirty patients had 1 readmission, 11 patients had 2 readmissions and 11 patients had 3 or more readmissions). The mean follow-up was 19 months with an overall and CSS of less than $20 \%$ (Table-2, Figure-2).

At the end of the follow-up, 8 patients (11\%) were alive, $47(65 \%)$ had died due to cancer, $7(9.7 \%)$ had died due to other causes, and $7(9.7 \%)$ were lost to follow-up.

\section{COMMENTS}

In the past, advanced age was a contraindication for cystectomy; some studies reported peri-operative mortality rates of $5.5 \%$ in patients younger than 60 , versus $11 \%$ in patients between 60 and 70 years old (4). Improvements in the surgical technique, anesthetic management and post-operative care have brought a reduction of major complications from an initial $51 \%$ down to the current $10-30 \%$, and the mortality rate subsequent to surgery has decreased to $2 \%$ (5), with transfusion, hospital stay, early and late complications and survival rates comparable to those of patients younger than 65 years. Moreover, patients presenting with an ASA score compatible with a major intervention are eligible for cystectomy because when it is compatible with major procedures, it reflects the functional status of a patient more accurately than age itself $(2,3)$.

Fifteen years ago, at our institution we only performed TUR. Later we started doing RC throughout the eighties, with two main objectives: improve 
local control disease with better oncological outcomes and minimize complications.
Regarding local control disease, the proportion of patients with advanced pathological stage

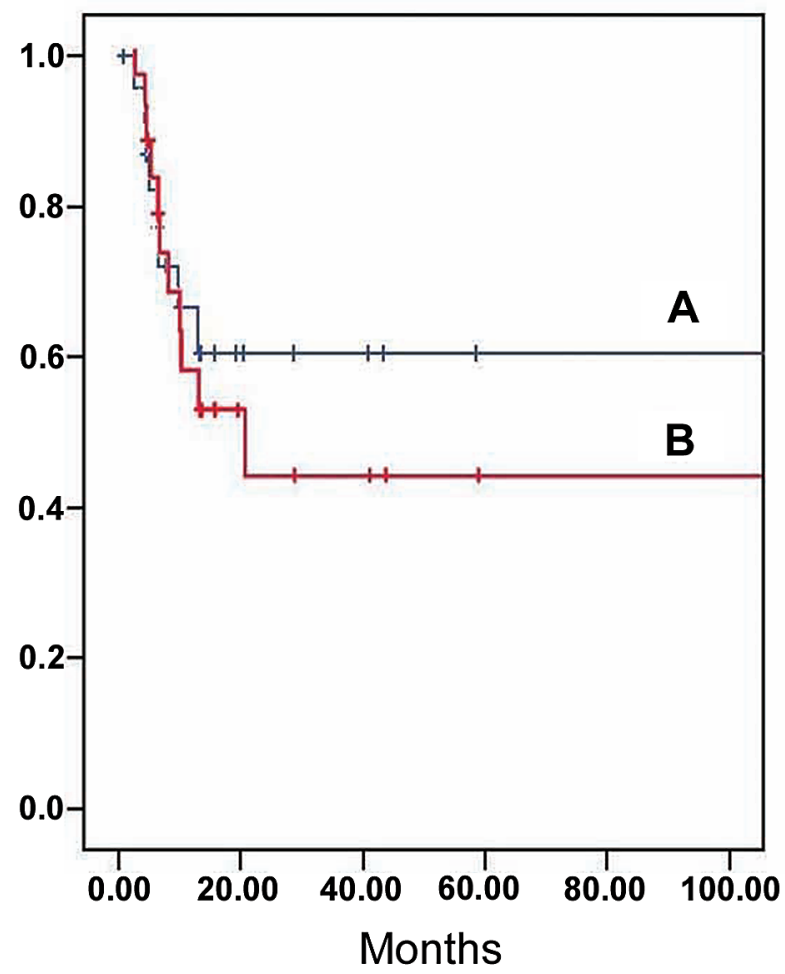

A : CSS $=60.54 \%$

$B: O S=42.9 \%$

Figure 1 - Survival rates in radical cystectomy patients: A) Cancer-specific survival (CSS), B) Overall survival (OS).

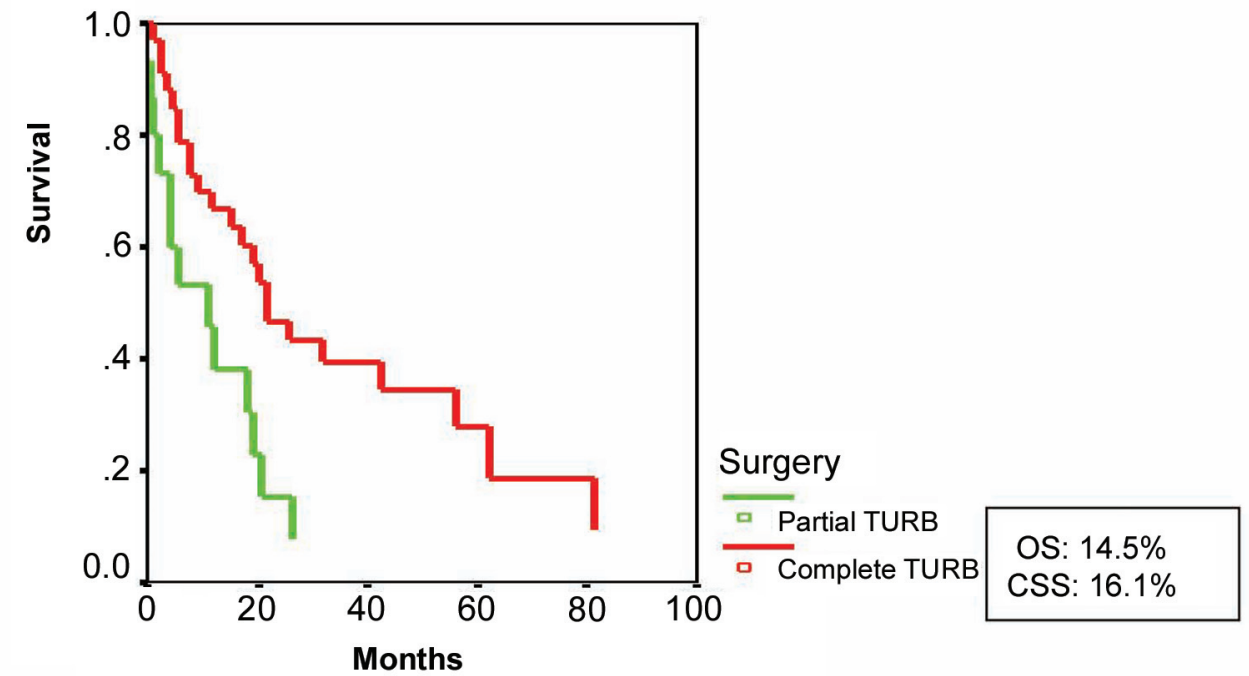

Figure 2 - Survival rates after partial or complete transurethral resection of bladder tumor (TURBT). 
Table 2 - Complications rate and oncological outcomes in RC and TUR.

\begin{tabular}{lcc}
\hline & Cystectomy & TUR \\
\hline Global complications rate (\%) & 33.2 & 11.1 \\
Transfusion (\%) & 37 & 13.8 \\
Follow-up (months) & 19 & 16.4 \\
Overall survival (\%) & 42.9 & 14.5 \\
Cancer specific survival (\%) & 60.5 & 16.1 \\
Readmission (\%) & 42 & 87.5 \\
\hline
\end{tabular}

$R C=$ radical cystectomy; $T U R=$ transurethral resection.

in our series was the highest one of all the series reviewed (81.48\%) (6-8); supporting the RC indication, since application of conservative alternatives (TUR) would yield a much poorer tumor control. Moreover, our $42.94 \%$ OS rate is similar to pT3 series published in younger population $(9,10)$ and the difference in elderly patient data, is because of a shorter follow-up. As to the CSS rate we obtained $60.54 \%$ and observed important differences at each stage. If we compare patients with pathologic stage pT2 to the rest of grouped stages, at our follow-up period, all of the pT2 patients continue to be alive, whereas survival of the rest of groups was $55.48 \%$. Most series reviewed report CSS rates between $62 \%$ and $84 \%$ (11-14) and no significant differences regarding OS or CSS were found in the series reviewed between patients younger and older than 70 years for RC $(15,16)$.

Comparing these results with transurethral resection of bladder tumor (TURBT) for MIBC, good results are shown in case of small T2 tumors $(17,18)$. Our survival rate is below $20 \%$, but we are dealing with not-selected patients with large tumor volumes ( $32 \%$ of them received only palliative TUR). At the end of the follow-up, only 8 patients (11\%) were alive and the OS rate was lower than $20 \%$ independently of the TUR being complete or partial (Figure-2). Therefore, compared to $\mathrm{RC}$ for this age group and with a similar follow-up, even if it is not a prospective and randomized study; overall and cancer-specific survivals are much more favorable for RC.

Regarding complications, intra-operative mortality in our series show two cases $(7.40 \%)$, whereas most studies reviewed, show rates between $0 \%$ and $7.40 \%$. The published series comparing intra operative mortality among patients younger and older than 70 shown no significant differences between both groups (15). Therefore, peri-operative mortality in the published RC series, some of them with 30 years of follow-up and with 1,359 patients with a mean 67 years of age, was only $2 \%$ (19); in this period the primary causes of mortality were those due to cardiovascular decompensation, sepsis frequently related to urinary or bowel fistula, pulmonary embolism and massive postoperative hemorrhage. In case of TUR we do not have any cases of intraoperative or perioperative mortality. The most frequent intraoperative complications were hemorrhage that was responsible for re-intervention in $84 \%$ of cases, perforation in $1.3 \%$ with incidence that is variable in the various series consulted (2.5-5\%) (20) and TUR syndrome.

Ten patients $(37 \%)$ required either intra- or post-operative blood transfusion, and the mean of transfused units was two (1-3). If differences between the mean units transfused and bleeding in patients younger and older than 70 are compared, significant differences between both groups regarding the mean bleeding in $\mathrm{mL}$ and in the number of transfused patients are found, even though transfused patients are larger in number in the older-than-70 group (9). In case of our TUR series, transfusion was necessary in 10 patients $(13.8 \%)$ that is lower compared with the $\mathrm{RC}$ group, but higher if it is compared with our global transfusion rate in TUR that is 3.4\% (21). 
The mean hospitalization stay of our patients in the group of RC was 16 days (range 8-28). Hospital stay varies between 7-34 days, and in our experience prolongation of hospital stay in patients older than 80 is related with the more difficult solution of their major complications. Moreover, patients with a poor ASA score will potentially be at greater risk of urinary derivation-related post-operative fistulae (2). In our series, the only patient that developed an entero-cutaneous fistula in the postoperative period had an ASA score of III.

TUR group reflect a mean hospitalization stay of seven days that is much better than the RC group, related to the lower impact of the endoscopic surgery for the patient. The problem with this group is a readmission rate that was $87 \%$, related in most cases with hematuria in the context of tumor persistence.

The evaluation of the risk-benefit ratio of the radio-chemotheraphy protocols in this cohort of patients over 80 with co-morbidities avoided their use. Available meta-analysis of randomized trials on cisplatin-containing combination neoadjuvant chemotherapy revealed a $5 \%$ difference in favor of neoadjuvant chemotherapy, and no randomized trials have yet compared survival with TURBT alone versus cystectomy for the management of patients with muscle-invasive disease, moreover collaborative international adjuvant chemotherapy trials are needed to assist researchers in assessing the true value of adjuvant chemotherapy (22).

Limitation of this study is that it was a descriptive, and not a comparative analysis of two independent series of patients. Further prospective randomized trials are necessary to confirm these findings.

\section{CONCLUSIONS}

$\mathrm{RC}$ in octogenarian patients is a safe procedure, with complication and survival rates comparable to those of the series of patients younger than 80 , and therefore we consider that age is not a contraindication of surgery if it is not accompanied by an unfavorable ASA score.

TUR for patients with MIBC within this age range is a much less morbid procedure, but disease specific survival is lower.

\section{CONFLICT OF INTEREST}

\author{
None declared.
}

\section{REFERENCES}

1. Ries LAG, Melbert D, Krapcho M, Stinchcomb DG, Howlader N, Horner MJ, Mariotto A, Miller BA, Feuer EJ, Altekruse SF, Lewis DR, Clegg L, Eisner MP, Reichman M, Edwards BK (Ed.), SEER Cancer Statistics Review, 1975-2005, National Cancer Institute. Bethesda, MD, http://seer.cancer.gov/csr/1975_2005/, based on November 2007 SEER data submission, posted to the SEER web site, 2008.

2. Malavaud B, Vaessen C, Mouzin M, Rischmann P, Sarramon J, Schulman C: Complications for radical cystectomy. Impact of the American Society of Anesthesiologists score. Eur Urol. 2001; 39: 79-84.

3. Wolters U, Wolf T, Stützer H, Schröder T: ASA classification and perioperative variables as predictors of postoperative outcome. Br J Anaesth. 1996; 77: 217-22.

4. Zincke $\mathrm{H}$ : Cystectomy and urinary diversion in patients eighty years old or older. Urology. 1982; 19: 139-42.

5. George L, Bladou F, Bardou VJ, Gravis G, Tallet A, Alzieu $\mathrm{C}$, et al.: Clinical outcome in patients with locally advanced bladder carcinoma treated with conservative multimodality therapy. Urology. 2004; 64: 488-93.

6. Soulié M, Straub M, Gamé X, Seguin P, De Petriconi $R$, Plante P, et al.: A multicenter study of the morbidity of radical cystectomy in select elderly patients with bladder cancer. J Urol. 2002; 167: 1325-8.

7. Chang SS, Alberts G, Cookson MS, Smith JA Jr: Radical cystectomy is safe in elderly patients at high risk. J Urol. 2001; 166: 938-41.

8. Clark PE, Stein JP, Groshen SG, Cai J, Miranda G, Lieskovsky G, et al.: Radical cystectomy in the elderly: comparison of clincal outcomes between younger and older patients. Cancer. 2005; 104: 36-43.

9. Kulovac B, Aganovic D, Prcic A: Radical cystectomy early postoperative complications and mortality rate. Med Arh. 2005; 59: 358-9.

10. Soloway MS, Lopez AE, Patel J, Lu Y: Results of radical cystectomy for transitional cell carcinoma of the bladder and the effect of chemotherapy. Cancer. 1994; 73: 1926-31.

11. Gamé X, Soulié M, Seguin P, Vazzoler N, Tollon C, Pontonnier F, et al.: Radical cystectomy in patients older than 75 years: assessment of morbidity and mortality. Eur Urol. 2001; 39: 525-9. 
12. Stroumbakis N, Herr HW, Cookson MS, Fair WR: Radical cystectomy in the octogenarian. J Urol. 1997; 158: 2113-7.

13. Ogawa A, Yanagisawa Y, Nakamoto T, Wajiki M, Hirabayashi N, Nakama M: Treatment of bladder carcinoma in patients more than 80 years old. J Urol. 1985; 134: 889-91.

14. Lodde M, Palermo S, Comploj E, Signorello D, Mian C, Lusuardi L, et al.: Four years experience in bladder preserving management for muscle invasive bladder cancer. Eur Urol. 2005; 47: 773-8; discussion 778-9.

15. Deliveliotis C, Papatsoris A, Chrisofos M, Dellis A, Liakouras C, Skolarikos A: Urinary diversion in highrisk elderly patients: modified cutaneous ureterostomy or ileal conduit? Urology. 2005; 66: 299-304.

16. May M, Fuhrer S, Braun KP, Brookman-Amissah S, Richter W, Hoschke B, et al.: Results from three municipal hospitals regarding radical cystectomy on elderly patients. Int Braz J Urol. 2007; 33: 764-73; discussion 774-6.

17. Solsona E, Iborra I, Ricós JV, Monrós JL, Dumont R: Feasibility of transurethral resection for muscle-infil- trating carcinoma of the bladder: prospective study. J Urol. 1992; 147: 1513-5.

18. Herr HW: Transurethral resection of muscle-invasive bladder cancer: 10-year outcome. J Clin Oncol. 2001; 19: 89-93.

19. Quek ML, Stein JP, Daneshmand S, Miranda G, Thangathurai D, Roffey $\mathrm{P}$, et al.: A critical analysis of perioperative mortality from radical cystectomy. J Urol. 2006; 175: 886-9; discussion 889-90.

20. Mydlo JH, Weinstein R, Shah S, Solliday M, Macchia $\mathrm{RJ}$ : Long-term consequences from bladder perforation and/or violation in the presence of transitional cell carcinoma: results of a small series and a review of the literature. J Urol. 1999; 161: 1128-32.

21. Collado A, Chéchile GE, Salvador J, Vicente J: Early complications of endoscopic treatment for superficial bladder tumors. J Urol. 2000; 164: 1529-32.

22. Calabrò F, Sternberg CN: Neoadjuvant and adjuvant chemotherapy in muscle-invasive bladder cancer. Eur Urol. 2009; 55: 348-58.
Accepted after revision: July 7, 2010

\section{Correspondence address: \\ Dr. Oscar Rodríguez Faba \\ C/ Cartagena, 340-350 \\ Barcelona, 08025, Spain \\ Fax: + 349 3416-9730 \\ E-mail: orodriguez@fundacio-puigvert.es}

\section{EDITORIAL COMMENT}

In the present manuscript, the authors compare the cancer specific outcomes of transurethral resection (TUR) versus radical cystectomy in octogenarians with muscle invasive bladder cancer. The authors address an interesting clinical question in a cohort we have traditionally associated to be at increased risk of perioperative complications with aggressive surgical intervention. The authors conclude appropriately that patients underlying (pre-treatment) co-morbidities (measured using ASA score) is more of a determinant of surgical risk than age alone. Similarly, the authors conclude that radical cystectomy offers an improved cancer specific survival (versus TUR) at the cost of increased perioperative morbidity. I would argue that 
TUR alone (without consideration of a bladder sparing type approach using chemo-radiotherapy or at the very least of adjuvant radiotherapy to the bladder and pelvic lymph nodes for local/regional cancer control) is not an oncologic efficacious treatment alternative except in the very rare case of a patient with a focally muscle invasive bladder tumor. Hence, the importance of repeat TUR (to ensure complete resection), random bladder biopsies (to rule out multifocal disease), and exam under anesthesia (to adequately assess clinical stage) should be considered essential in these select patients treated by TUR alone. Similarly, careful pathologic review of the TUR specimen by an experi- enced genitourinary pathologist is essential to confirm tumor invasion into the muscularis propria and rule out the presence of adverse pathological features such as lymphovascular invasion or micropapillary histology.

In summary, there clearly remains a subset of patients with muscle invasive bladder cancer for whom cystectomy is not a feasible choice because of pre-operative co-morbidities or by their refusal to proceed with aggressive surgical intervention. In these patients, clinicians must tailor their treatment options based on patient and tumor specific characteristics.

Dr. Philippe E. Spiess Department of Interdisciplinary Oncology Moffitt Cancer Center University of South Florida Tampa, Florida, USA E-mail:Philippe.Spiess@moffitt.org 